ISAHP Article: Sensitivity analysis for the assessment of adaptive capacity to climate change: a case of study form Brazil. To Be Submitted to the International Symposium of the Analytic Hierarchy Process 2020, Web Conference.

\title{
SENSITIVITY ANALYSIS FOR THE ASSESSMENT OF ADAPTIVE CAPACITY TO CLIMATE CHANGE: A CASE OF STUDY FROM BRAZIL
}

Ileana Grave, Luis A. Bojórquez-Tapia, Alejandra Estrada-Barón, Donald R Nelson, Hallie Eakin

\begin{abstract}
While the Analytic Hierarchy Process (AHP) has been widely used to ascertain the vulnerability of households to environmental change, the sensitivity of adaptive capacity indices to uncertain appraisals and judgments of the magnitudes and weights of indicators has been largely ignored so far. In this work, we present an approach to identify the most influential indicators of household adaptive capacity. The approach entails the implementation of two sensitivity analyses: indicator removal and threshold value. The adaptive capacity indicators were selected and ranked for household surveys carried out in Brazil in 1998 and 2012.
\end{abstract}

Keywords: AHP, multicriteria, vulnerability, household, Latin America

\section{Introduction}

Building the adaptive capacity of households to climate change has become a high policy priority. The AHP has been widely used to develop indicator-based metrics to ascertain the adaptive capacity of households (Eakin and Bojórquez-Tapia, 2008). By means of the AHP, a set of households can be ranked in terms of a set of incommensurable criteria that describe the vulnerability and adaptive capacity. Indicator based metrics are critical components of poverty reduction and climate risk management strategies and interventions. However, such rankings can be problematic due to the uncertainty involved in the appraisal of the criteria magnitudes and weights. Despite that this may result in unknown errors, evaluating the sensitivity of vulnerability indices to that uncertainty has been largely ignored.

In this paper, we present an approach to evaluate the sensitivity of household rankings to the uncertainty of both criteria weights and criteria magnitudes used in adaptive capacity indices. Our approach draws from Triantaphyllou (Triantaphyllou and Sánchez, 1997) to identify the most critical indicators. It entails the implementation of two tests: indicator removal and threshold value.

We illustrate our approach using data from a longitudinal research design to explore the role of anti-poverty conditional cash transfer programs in building adaptive capacity in the state of Ceará, Brazil. Our results show that the adaptive capacity indicators or criteria with the higher weights are not necessarily the ones that denote the most important changes in the household rankings. We concluded that sensitivity analysis is fundamental to understand the effectiveness of government programs to address climate change vulnerability and adaptive capacity. 
ISAHP Article: Sensitivity analysis for the assessment of adaptive capacity to climate change: a case of study form Brazil. To Be Submitted to the International Symposium of the Analytic Hierarchy Process 2020, Web Conference.

\section{Methodology}

The adaptive capacity index is developed using the AHP for household surveys carried out in 1998 and 2012. Following Eakin and Bojórquez-Tapia (2008), the respective indicators are organized into hierarchies (Fig. 1). For each hierarchy, top-level corresponded to the overall goal (to rank households in terms of their vulnerability related to either adaptive capacity), intermediate levels corresponded to the set of criteria and indicators to specify the overall goal, and the lowest level to the alternatives (households). For each level, a pairwise comparison matrix is generated to elicit the relative weights for each indicator. Given a hierarchy, the elements at each level are weighted according to their importance on the level above.

The database of households encompassed the surveys carried out in $1998(n=484)$ and $2012(n=477)$, and 18 indicators, five of which were not measured in 1998 (saving accounts, health access, participation, frequency of support given and crop insurance) and one was not measured in 2012 (hora de plantar). Because the number of indicators differed between surveys, the global importance weights are rescaled linearly setting to zero the weights for the missed indicators.

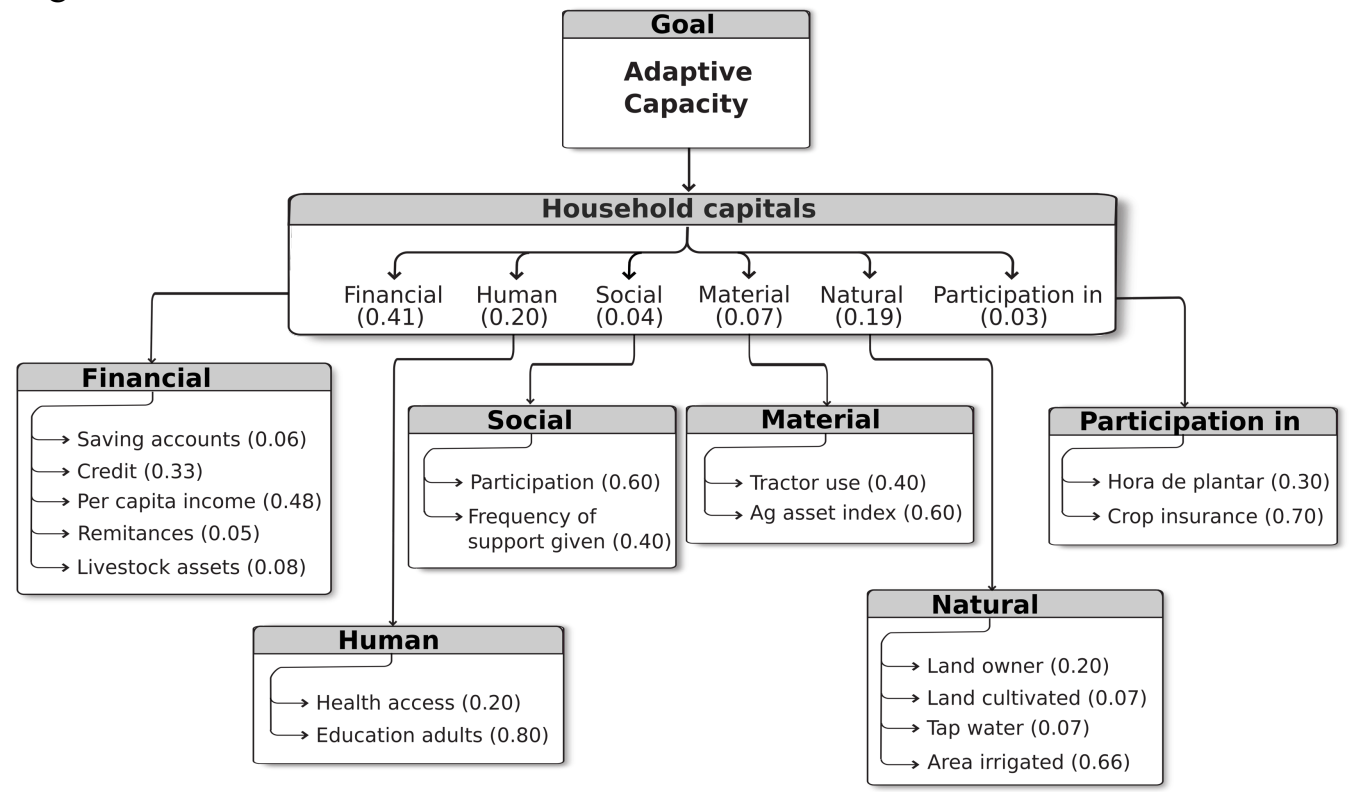

Figure 1. AHP model for the Adaptive Capacity index

A score $V$, of each household is computed by aggregating the proper criteria weights and normalized scores of indicators using a weighted linear combination:

$$
V_{i}^{h}=\sum_{j}^{J} w_{i j} x_{i j}^{h}
$$

where $w$ is the weight of an indicator, $x$ is the standardized score, and $h, i$, and $j$ indicate household, vulnerability index and vulnerability indicator, respectively.

The criteria weights, $w$, are elicited through the AHP, whereas the standardized scores, $x$, are obtained by means of value functions. The value functions are used to transform the natural scale of indicators (in different scales and units) into a uniform [0,1] scale with ratio properties, 0 representing the most undesirable state and 1 the most desirable state 
ISAHP Article: Sensitivity analysis for the assessment of adaptive capacity to climate change: a case of study form Brazil. To Be Submitted to the International Symposium of the Analytic Hierarchy Process 2020, Web Conference.

(Beinant, 1997). The value functions reflected the assumption that vulnerability is higher as adaptive capacity decreases; they accounted for the possibility of nonlinear or nonmonotonic relationships between natural and value scales.

Indicator removal test. The indicator removal test involved setting to zero the weight for indicator $r\left(w_{\mathrm{ir}}=0\right)$, and rescaling the remaining indicator weights linearly $\left(w_{\mathrm{ij}}^{\prime}=w_{\mathrm{ij}} \div\right.$ $\sum_{j \neq r}^{J} w_{\mathrm{ij}}$ ). Then Eq. (1) is applied to generate the respective household vulnerability index $\left(V_{i j \neq r}^{h}=\sum_{j \neq r}^{J} w_{\mathrm{ij}}^{\prime} x_{\mathrm{ij}}^{h}\right)$. The change (in percentage) of the median vulnerability by the removal of the $r$-th indicator is obtained as

$$
V_{\mathrm{ir}}^{\Delta Q_{2}}=\left|\frac{V_{i j \neq r}^{Q_{2}}-V_{i}^{Q_{2}}}{V_{i}^{Q_{2}}}\right| \times 100
$$

where $V_{i j \neq r}^{Q_{2}}$ is the median vulnerability by the removal of the $r$-th indicator and $V_{i}^{Q_{2}}$ is the median vulnerability of all the indicators.

Threshold value test. The threshold value test is based on the concept of the probability of "rank reversal" between the vulnerability scores of a household $V_{i}^{h}$, and some reference value $V_{i}^{\rho}$, resulting from changing the standardized scores $x$, so that if $V_{i}^{\rho} \geq V_{i}^{h}$ swaps to $V_{i}^{\rho}<V_{i}^{h}$, or if $V_{i}^{\rho} \leq V_{i}^{h}$ swaps to $V_{i}^{\rho}>V_{i}^{h}$. Following the methodology presented by Triantaphyllou (Triantaphyllou, 1997) the change required to generate a rank reversal

$$
\tau_{i j}^{h}=\frac{V_{i}^{h}-V_{i}^{\rho}}{w_{i j}}
$$

is calculated with respect to the median vulnerability as the reference value $V_{i}^{\rho}$. In particular, the feasibility of change is conditioned to the range $x_{i j}^{h}-1 \leq \tau_{i j}^{h} \leq x_{i j}^{h}$ to avoid changes $\bar{x}_{i j}^{h}=x_{i j}^{h}-\tau_{i j}^{h}$ of a standardized score $x_{i j}^{h}$ to be outside the standardized value $[0,1]$.

Finally, the critical indicator value $\mathcal{C}_{i j}$ of an indicator, $j$, is obtained by the product of the probability $p_{i j}$ of rank reversals and the sensitivity coefficient

$$
s_{\mathrm{ij}}=\frac{1}{\Delta_{i j}},
$$

where $\Delta_{i j}=\left|\tau_{i j}^{h}\right|^{Q_{m}}$ is taken for a particular quantile $Q_{m}$. Thus, the higher the frequency and magnitude of rank reversals, the higher the criticality of a criterion. It is important to notice that when $V_{i}^{h}=V_{i}^{\rho}$ the sensitivity coefficient goes to infinity, in this case, $\Delta_{i j}$ is approximated with 0.001 .

\section{Results}

For the indicator removal test the most critical indicators for the adaptive capacity index were found to be: land owner, education adults and per capita income (1998 survey), and credit and education adults (2012 survey). Because the number of indicators differed, test (2) was applied for each survey.

For the threshold value test, the critical indicator value $\mathcal{C}_{i j}$ was calculated considering two types of quartile measures: i) the first $Q_{1}$ and the ii) second $Q_{2}$ or median. In the following, 
ISAHP Article: Sensitivity analysis for the assessment of adaptive capacity to climate change: a case of study form Brazil. To Be Submitted to the International Symposium of the Analytic Hierarchy Process 2020, Web Conference.

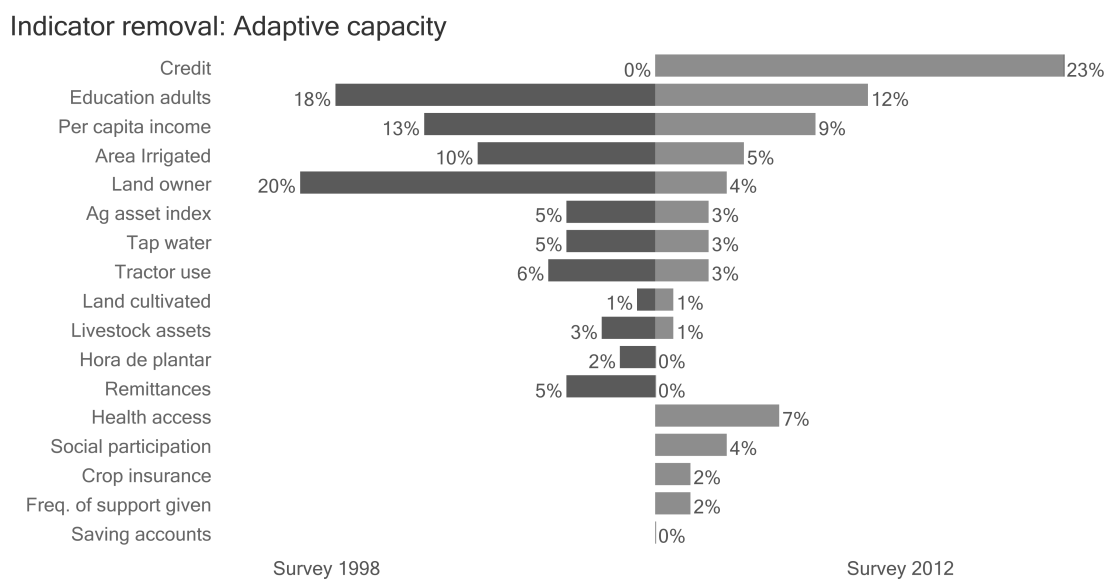

Figure 2. Results of the indicator removal test

results for both measures and surveys are presented and compared. For both quartiles and surveys, results of the threshold value test classified the indicators in terms of criticality (Figure 4) as follows: high, per capita income; moderate, credit, education adults and area irrigated and low, ag asset index, livestock assets, landowner, tractor use, remittances, land cultivated and tap water. A comparison between surveys showed a decrease in the critical indicator value between 1998 and 2012 for the most sensitive adaptive capacity indicators.

\section{Discussion and Conclusions}

As was previously stated, to identify the most influential vulnerability indicators for household rankings, it is important to consider the uncertainty involved in the magnitudes and weights of the standardized scores of vulnerability indices.

Results suggest that vulnerability indicators that were ranked as the most important were not necessarily the most influential and that changes generated by certain indicators cannot be ignored. Moreover, even when different statistical measures can be considered to capture the "common" characteristics of the area of study, the most influential indicators are preserved.

Considering the case of the most influential indicators, our results showed that the most important indicators for the adaptive capacity index were per capita income, education adults and credit in both surveys (1998 and 2012). Intuition says that these should be the most sensitive indicators, however, as the indicator removal test showed, the most critical indicators were land owner, education adults and per capita income, for the 1998 survey, and credit and education adults, for the 2012 survey. It is important to notice that, in this particular case, the most critical indicator in the 1998 survey is one with medium importance (land owner). Our results substantiate the assertion by Triantaphyllou (1997) that the most sensitive criterion may be the one with the lowest weight.

The difference between survey indicators suggested the inclusion of new public policies by the government, this fact is reflected in the change in magnitude of specific indicators. For example, the introduction of the indicator soil quality in 2012 surveys caused a 
ISAHP Article: Sensitivity analysis for the assessment of adaptive capacity to climate change: a case of study form Brazil. To Be Submitted to the International Symposium of the Analytic Hierarchy Process 2020, Web Conference.

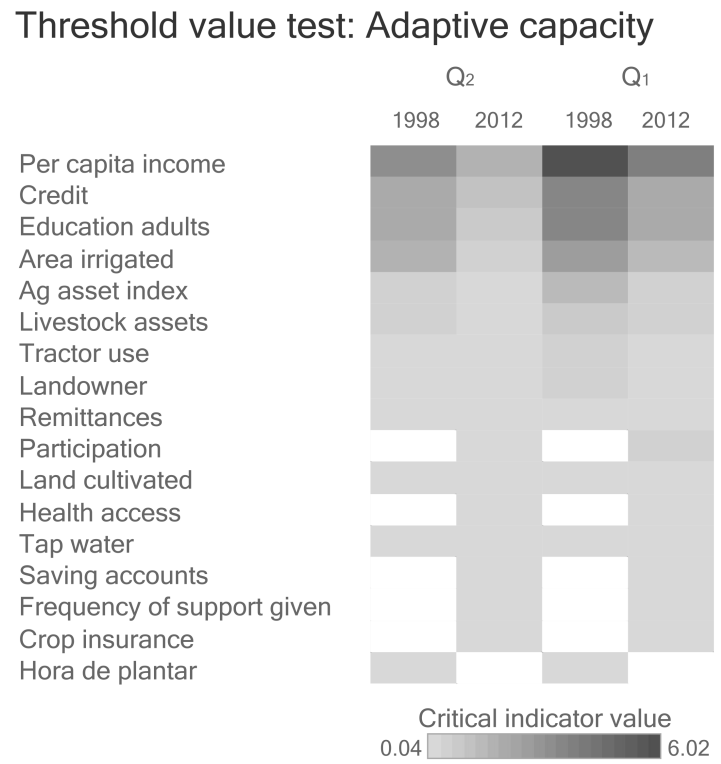

Figure 3. Results of the threshold value test: first quartile $Q_{1}$ and median $Q_{2}$

significant reduction on the critical indicator value of the most sensitive indicators, in particular, the indicators household pays rent and \% income ag sales which were classified in the most critical group, changed to the moderately critical indicators group.

One of the principal limitations of the techniques presented in this work is the fact that, even when the uncertainty in the judgments and magnitude evaluation is considered, only indices defined as linear combinations can be analyzed. However, it is important to emphasize that this also gives a wide field of applications in problems related to land suitability, environmental impact assessment and socio-ecological vulnerability indicators.

Acknowledgments. This work was supported by UNAM PAPIIT-IV100118; ASUUNAM Binational Laboratory of Sustainability, Vulnerability, and Adaptation to Climate Change; FOMIX YUC-2018-04-01-88958.

\section{Key References}

Eakin, H. \& Bojorquez-Tapia, L. (2008). "Insights into the composition of household vulnerability from multicriteria decision analysis," Global Environmental Change, vol. 18, no. 1, pp. 112-127, 2.

Saaty, T. (1980). The Analytic Hierarchy Process. McGraw-Hill, New York.

Triantaphyllou, E. \& A. Sánchez. (1997) "A Sensitivity Analysis Approach for Some Deterministic Multi-Criteria Decision-Making Methods," Decision Sciences, vol. 28, no. 1, pp. 151-194.

Beinat, E. (1997) Value Functions for Environmental Management. Springer Netherlands, vol. 7. 